\section{ORIGINAL RESEARCH}

\author{
J. Hagemeier \\ B. Weinstock-Guttman \\ N. Bergsland \\ M. Heininen-Brown \\ E. Carl \\ C. Kennedy \\ C. Magnano \\ D. Hojnacki \\ M.G. Dwyer \\ R. Zivadinov
}

\title{
Iron Deposition on SWI-Filtered Phase in the Subcortical Deep Gray Matter of Patients with Clinically Isolated Syndrome May Precede Structure-Specific Atrophy
}

BACKGROUND AND PURPOSE: Increasing evidence suggests that iron deposition is present in the later stages of MS. In this study we examined abnormal phase values, indicative of increased iron content on SWI-filtered phase images of the SDGM in CIS patients and HC. We also examined the association of abnormal phase with conventional MR imaging outcomes at first clinical onset.

MATERIALS AND METHODS: Forty-two patients with CIS (31 female, 11 male) and 65 age and sexmatched HC (41 female, 24 male) were scanned on a 3T scanner. Mean age was 40.1 (SD = 10.4) years in patients with $\mathrm{CIS}$, and $42.8(\mathrm{SD}=14)$ years in $\mathrm{HC}$, while mean disease duration was 1.2 years $(\mathrm{SD}=1.3)$ in patients with CIS. MP-APT, NPTV, and normalized volume measurements were derived for all SDGM structures. Parametric and nonparametric group-wise comparisons were performed, and associations were determined with other MR imaging metrics.

RESULTS: Patients with CIS had significantly increased MP-APT $(P=.029)$ and MP-APT volume $(P=$ $.045)$ in the pulvinar nucleus of the thalamus compared with HC. Furthermore, the putamen $(P=.004)$, caudate $(P=.035)$, and total $\operatorname{SDGM}(P=.048)$ displayed significant increases in MP-APT volume, while MP-APT was also significantly increased in the putamen $(P=.029)$. No global or regional volumetric MR imaging differences were found between the study groups. Significant correlations were observed between increased MP-APT volumes of total SDGM, caudate, thalamus, hippocampus, and substantia nigra with white matter atrophy and increased T2 lesion volume $(P<.05)$.

CONCLUSION: Patients with CIS showed significantly increased content and volume of iron, as determined by abnormal SWI-phase measurement, in the various SDGM structures, suggesting that iron deposition may precede structure-specific atrophy.

\begin{abstract}
ABBREVIATIONS: $\mathrm{CIS}=$ clinically isolated syndrome; EDSS = Expanded Disability Status Scale; $\mathrm{ETL}=$ echo-train length; FIRST = fMRI-integrated registration and segmentation tool; $\mathrm{Gd}=$ gadolinium; $\mathrm{GM}=$ gray matter; $\mathrm{HC}=$ healthy controls; $\mathrm{LV}=$ lesion volume; MP-APT = mean phase of the abnormal phase tissue; NBV = normalized brain volume; NGMV = normalized gray matter volume; NLVV = normalized lateral ventricle volume; NPTV = normal phase tissue volume; NWMV = normalized white matter volume; $\mathrm{PFOV} \mathrm{=} \mathrm{phase} \mathrm{FOV;} \mathrm{RRMS} \mathrm{=} \mathrm{relapsing-remitting} \mathrm{MS;}$ SDGM = subcortical deep GM
\end{abstract}

I thas become increasingly clear that GM damage is present in patients with MS and involves both the cortical GM and SDGM structures. ${ }^{1}$ GM damage is most extensive in patients with progressive $\mathrm{MS},{ }^{2}$ but is already present in those with $\mathrm{CIS}^{3-10}$ and can predict conversion to clinically definite MS. ${ }^{11-13}$

Most authors investigating iron deposition in MS have used imaging techniques such as T2 hypointensity, ${ }^{14-16}$ relaxometry, ${ }^{17-19}$ magnetic field correlation, ${ }^{20}$ and SWI. ${ }^{21-23}$ Accumulation of iron in the SDGM has also been observed histologically in $\mathrm{MS}^{24,25}$ and iron deposition has been detected in the same SDGM structures where the volume loss also oc-

Received October 31, 2011; accepted after revision November 23.

From the Buffalo Neuroimaging Analysis Center (J.H., N.B., M.H.-B., E.C., C.K., C.M., M.G.D., R.Z.) and The Jacobs Neurological Institute (B.W.-G., D.H., R.Z.), State University of New York at Buffalo, Buffalo, New York.

Please address correspondence to Robert Zivadinov, MD, PhD, FAAN, Department of Neurology, School of Medicine and Biomedical Sciences, Buffalo Neuroimaging Analysis Center, 100 High St., Buffalo, New York 14203; e-mail: rzivadinov@bnac.net

Indicates open access to non-subscribers at www.ajnr.org

http://dx.doi.org/10.3174/ajnr.A3030 curred. ${ }^{23}$ It is thought that excessive levels of iron in the brain may lead to tissue damage by the generation of reactive oxygen species, most probably through the Fenton reaction. ${ }^{26,27}$ In this process, ferrous iron $\left(\mathrm{Fe}^{2+}\right)$ donates an electron to hydrogen peroxide, with the resulting toxic products being ferric iron $\left(\mathrm{Fe}^{3+}\right)$, hydroxyl anion $\left(\mathrm{OH}^{-}\right)$, and the highly reactive hydroxyl radical $(\mathrm{OH})$.

Few studies have investigated iron deposition in the earliest stages of MS, such as in those with CIS. ${ }^{28}$ Ceccarelli et $\mathrm{al}^{29}$ reported increased T2 hypointensity in the head of the left caudate nucleus of patients with CIS compared with HC. However, a recent study using relaxometry did not show differences between patients with CIS and HC. ${ }^{19}$ Moreover, patients with RR-MS appear to show even higher iron content in the SDGM than patients with CIS, suggesting that disease progression may be related to pronounced iron deposition. ${ }^{18,19,30}$ If increased levels of iron are causally related to SDGM damage and disease development, iron deposition must be present at the earliest stages of the disease, such as in patients with CIS.

This study used a SWI-filtered phase approach to characterize possible iron deposition in the SDGM of patients with CIS. Paramagnetic substances within the brain, mostly in the 
form of ferritin, influence the frequency of proton spin and cause local magnetic field changes. ${ }^{31}$ The SWI-filtered phase imaging method uses a complex-space high-pass filter to retain only biologically relevant phase shifts, to generate a metric of iron attenuation. ${ }^{23,31}$

In this study, we compared SDGM measurements of abnormal phase, indicative of increased iron content, and normalized volume between patients with CIS and a group of $\mathrm{HC}$. Moreover, we sought to investigate the relationship of these measurements with conventional MR imaging techniques.

\section{Materials and Methods}

\section{Subjects}

We prospectively enrolled 42 consecutive patients with CIS (31 women, $11 \mathrm{men}$ ). Inclusion criteria were a diagnosis of CIS, ${ }^{32}$ age $18-65$ years, and disease duration between 0 and 5 years. Sixty-five age- and sex-matched HC were also prospectively enrolled, all of whom had normal MR imaging and physical examinations. Patients with CIS underwent full neurologic assessment, including determination of EDSS. ${ }^{33}$ Participants were excluded if they had a relapse or were treated with steroids within the month preceding study entry, were pregnant, or had any pre-existing medical conditions known to be associated with brain pathology. The internal institutional review board approved the study protocol and written informed consent was obtained from all participants.

\section{Image Acquisition}

A 3T Signa Excite HD 12.0 (GE Healthcare, Milwaukee, Wisconsin) with an 8-channel head and neck (HDNV) coil was used for scanning purposes. SWI data were acquired using a 3D flow-compensated gradient recalled-echo sequence with 64 locations, $2 \mathrm{~mm}$ thickness, a $512 \times 192$ (frequency $\times$ phase) matrix, $\mathrm{FOV}=25.6 \mathrm{~cm} \times 19.2 \mathrm{~cm}$, with $\mathrm{pFOV}=0.75$, resulting in an in-plane resolution of $0.5 \mathrm{~mm} \times 1$ $\mathrm{mm}$. Other relevant parameters included flip angle $=12^{\circ}, \mathrm{TE}=22$, $\mathrm{TR}=40 \mathrm{~ms}$, and acquisition time $=8 \mathrm{~min}, 46 \mathrm{s.}^{23}$

In addition, we acquired the following sequences: $2 \mathrm{D}$ multiplanar dual FSE proton attenuation and T2-weighted imaging (TE1/TE2/ $\mathrm{TR}=9 / 98 / 5300 \mathrm{~ms}$, flip angle $=90^{\circ}$, and ETL $=14 ;$ FLAIR $(\mathrm{TE} / \mathrm{TR}=$ $120 / 8500 \mathrm{~ms}, \mathrm{TI}=2100 \mathrm{~ms}$, flip angle $\left.=90^{\circ}, \mathrm{ETL}=24\right)$; 3D highresolution T1WI using fast-spoiled gradient echo with magnetization-prepared inversion recovery pulse (TE/TI/TR $=2.8 / 900 / 5.9 \mathrm{~ms}$, flip angle $\left.=10^{\circ}\right)$; and spin-echo T1WI $(\mathrm{TE} / \mathrm{TR}=16 / 600 \mathrm{~ms}$, flip angle $=90^{\circ}$ ). All sequences except SWI were obtained with a $256 \times$ 192 matrix, $\mathrm{FOV}=25.6 \mathrm{~cm} \times 19.2 \mathrm{~cm}, \mathrm{pFOV}=0.75$, for an in-plane resolution of $1 \mathrm{~mm} \times 1 \mathrm{~mm}$. Forty-eight sections were collected, with a thickness of $3 \mathrm{~mm}$, and no gap between sections, for all 2D scans (proton attenuation/T2, FLAIR, and spin-echo T1). For the 3D highresolution inversion recovery fast-spoiled gradient echo, 184 1-mmthick locations were acquired, resulting in isotropic resolution. All scans were prescribed in an axial-oblique orientation, parallel to the subcallosal line. One average was used for all sequences.

\section{Image Analyses}

Analyses were performed by operators who were unaware of the participants' disease status.

\section{Abnormal Phase Identification}

Raw ( $k$-space) SWI data were transferred to an off-line Linux workstation for postprocessing using software developed in-house and written in Matlab (MathWorks, Natick, Massachusetts). A detailed overview of SWI image acquisition, processing, analysis, and validation is provided elsewhere. ${ }^{23}$

To segment SDGM structures, a combination of semiautomated edge-contouring and FMRIB's FIRST (http://www.fmrib.ox.ac.uk/ fsldownloads/) on 3D T1 WI was used. ${ }^{34}$ Specifically, the thalamus, caudate, putamen, globus pallidus, hippocampus, amygdala, and nucleus accumbens were identified in this way. ${ }^{35}$ Structures not identifiable by FIRST, such as the red nucleus, pulvinar nucleus of the thalamus, and substantia nigra, were identified semiautomatically using JIM5 (Xinapse Systems, Northamptonshire, United Kingdom) on the most representative section for each subject. ${ }^{35}$

Using the SWI-filtered phase images, additional processing was performed to identify voxels likely to contain abnormal amounts of iron, based on their phase values. Reference phase values (means and $\mathrm{SD}$ ) for each SDGM structure were determined previously using a large sample of HC. ${ }^{23}$ Only the voxels of SDGM structures with phase values lower than 2 standard deviations below the reference group were retained. This yielded structure-specific maps of voxels with abnormally low phase. Subsequently, as a measure of the level of phase decrease, the mean value of subthreshold voxels was calculated to yield the MP-APT. More negative MP-APT values indicate increased iron content within that region, and mean values are reported in radians. In addition, we determined the SDGM NPTV by subtracting the abnormal phase tissue volume of a particular structure from the total volume of the same structure.

\section{Global Atrophy and Lesion Analyses}

The SIENAX cross-sectional software tool (version 2.6; http://www. fmrib.ox.ac.uk/fsl/feeds/doc/index.html) was used for brain extraction and tissue segmentation, with correction for T1-hypointensity misclassification. ${ }^{36}$ As described previously,${ }^{37}$ we acquired the following volume measures: NBV, NGMV, NWMV, and NLVV. T2- and Gd-LVs and numbers were measured using a semiautomated edge detection contouring/thresholding technique, as described previously. ${ }^{38}$ Normalized volumes were obtained for all SDGM structures using FIRST. ${ }^{35}$

\section{Statistical Analysis}

Analyses were conducted using PASW Statistics, version 18.0 (IBM, Somers, New York). Differences between the groups were tested using the $\chi^{2}$-test (sex) and Student $t$ test. Distributions of the data were tested for normality using the Shapiro-Wilk test. Because SDGM MPAPT, MP-APT volume, and NPTV were not normally distributed $(P<.001)$, the Mann-Whitney $U$ test was used to evaluate group differences of these variables. SDGM volume measurements were compared using the Student $t$ test. Effect sizes are stated as Cohen $d$. We used Spearman correlation coefficients to assess the relationship between SDGM MP-APT, MP-APT volume, NPTV, and normalized volume with other MR imaging variables. Nominal $P$ values $<.05$ were regarded as significant, using 2 -tailed testing, and $P$ values $<.10$ were considered a trend.

\section{Results}

Table 1 shows the demographic, clinical, and MR imaging characteristics of patients with CIS and HC. The mean age of patients with CIS was 40.1 years $(S D=10.4)$, with a mean disease duration of 1.2 years $(\mathrm{SD}=1.3)$ and median EDSS score of 1.0 (range $=0-2.5)$. Thirty-one $(73.8 \%)$ patients with CIS were female. Of the 42 patients with CIS, 22 (52.4\%) were 
Table 1: Demographic, clinical, and MRI characteristics of the study groups

\begin{tabular}{|c|c|c|c|}
\hline & $\begin{array}{c}\mathrm{HC} \\
(n=65)\end{array}$ & $\begin{array}{c}\text { CIS } \\
(n=42)\end{array}$ & $\begin{array}{c}P \\
\text { value }\end{array}$ \\
\hline Female ( $n[\%])$ & $41(63.1)$ & $31(73.8)$ & .295 \\
\hline Age in years (mean $[S D]$ ) & $42.8(14)$ & $40.1(10.4)$ & .288 \\
\hline Age at onset in years (mean [SD]) & NA & $38.8(10.6)$ & NA \\
\hline $\begin{array}{l}\text { Disease duration in years (mean }[\mathrm{SD}] \\
\text { median) }\end{array}$ & NA & $1.2(1.3) 1$ & NA \\
\hline EDSS (median [range]) & NA & $1.0(0-2.5)$ & NA \\
\hline $\begin{array}{l}\text { Presence of disease-modifying therapy } \\
\text { Type of onset }(n[\%])\end{array}$ & NA & $22(52.4 \%)$ & NA \\
\hline Polysymptomatic & NA & $7(16.7 \%)$ & \\
\hline Monosymptomatic & NA & $35(83.3 \%)$ & \\
\hline Spinal cord syndrome & & $23(65.7 \%)$ & \\
\hline Optic neuritis & & $8(22.9 \%)$ & \\
\hline Brain stem/cerebellar & & $4(11.4 \%)$ & \\
\hline Gd lesion number (mean [SD]) & NA & $.4(1.1)$ & NA \\
\hline T2 lesion number (mean [SD]) & NA & $12.3(15.3)$ & NA \\
\hline 1-4 lesions (n [\%]) & & $15(35.7 \%)$ & \\
\hline 5-8 lesions ( $n[\%])$ & & $6(14.3 \%)$ & \\
\hline$\geq 9$ lesions ( $n[\%])$ & & $18(42.9 \%)$ & \\
\hline Gd lesion volume (mean [SD]) & NA & $.08(.3)$ & NA \\
\hline T2 lesion volume (mean [SD]) & NA & $1.7(2.6)$ & NA \\
\hline NGMV (mean [SD]) & $822.3(63.2)$ & $821.5(58.2)$ & .953 \\
\hline NWMV (mean [SD]) & $775.3(63.3)$ & $760.6(40.2)$ & .154 \\
\hline NBV (mean [SD]) & $1592.9(99.6)$ & $1583(56.5)$ & .521 \\
\hline NLVV (mean [SD]) & $27.9(13.7)$ & 32.6 (18.9) & .145 \\
\hline
\end{tabular}

Note:-All lesion and brain volumes are expressed in milliliters. Of the 42 patients with CIS, 33 presented with brain T2 lesions and 9 with spinal cord T2 lesions. Differences between the groups were tested using the $\chi^{2}$ (gender) test and Student $t$ test. NA indicates not available.

\begin{tabular}{|c|c|c|c|c|c|}
\hline & \multicolumn{2}{|c|}{ MP-APT } & \multirow[b]{2}{*}{$d$} & \multirow{2}{*}{$\begin{array}{c}\% \\
\text { Difference }\end{array}$} & \multirow{2}{*}{$\begin{array}{c}P \\
\text { Value }\end{array}$} \\
\hline & $\mathrm{HC}$ & $\mathrm{CIS}$ & & & \\
\hline Total SDGM & $-.143(.019)$ & $-.15(.024)$ & .31 & 5 & .171 \\
\hline Caudate & $-.165(.024)$ & $-.171(.013)$ & .31 & 3.6 & .140 \\
\hline Putamen & $-.169(.036)$ & $-.19(.045)$ & .5 & 12.4 & .029 \\
\hline Globus pallidus & $-.177(.04)$ & $-.174(.02)$ & .11 & 1.7 & .652 \\
\hline Thalamus & $-.09(.017)$ & $-.096(.012)$ & .37 & 6.7 & .067 \\
\hline Hippocampus & $-.158(.046)$ & $-.151(.037)$ & .16 & 4.4 & .329 \\
\hline Amygdala & $-.2(.070)$ & $-.209(.074)$ & .14 & 4.5 & .632 \\
\hline Nucleus accumbens & $-.72(.187)$ & $-.794(.253)$ & .33 & 10.3 & .581 \\
\hline Red nucleus & $-.228(.036)$ & $-.235(.024)$ & .22 & 3.1 & .422 \\
\hline Substantia nigra & $-.303(.056)$ & $-.313(.042)$ & .22 & 3.3 & .381 \\
\hline Pulvinar & $-.135(.022)$ & $-.148(.019)$ & .61 & 9.6 & .004 \\
\hline
\end{tabular}

Note:-MP-APT is expressed in radians. Differences between groups were tested using Mann-Whitney $U$ test.

on disease-modifying therapy and 35 (83.3\%) had a monosymptomatic onset. No significant differences were observed in age, sex, or atrophy measures between patients with CIS and HC. Of the 42 patients with CIS, 33 presented with brain T2 lesions and 9 with spinal cord T2 lesions. Of those patients with CIS who had brain lesions, 18 (54.5\%) showed $\geq 9$ T2 hyperintense lesions, and 4 patients showed Gd-active lesions.

Table 2 displays MP-APT differences in SDGM structures between $\mathrm{HC}$ and patients with CIS. Both the pulvinar nucleus of the thalamus $(P=.004)$ and putamen $(P=.029)$ showed increased MP-APT, and there was a trend for increased MPAPT in the thalamus $(P=.067)$. These SDGM structures showed an increase of $9.6 \%, 12.4 \%$, and $6.7 \%$ in MP-APT, respectively.
Table 3: Differences in MP-APT volume between patients with CIS and $\mathrm{HC}$

\begin{tabular}{lcccccc}
\hline & \multicolumn{2}{c}{ MP-APT Volume } & & \multicolumn{2}{c}{$\%$} & $P$ \\
\cline { 2 - 3 } & HC & CIS & & $d$ & Difference & Value \\
\hline Total SDGM & $14.33(2.07)$ & $15.21(1.96)$ & .44 & 6.1 & .048 \\
Caudate & $2.33(.54)$ & $2.6(.59)$ & .5 & 11.6 & .035 \\
Putamen & $2.64(.8)$ & $3.1(.79)$ & .58 & 17.4 & .004 \\
Globus pallidus & $1.33(.25)$ & $1.37(.29)$ & .16 & 3 & .396 \\
Thalamus & $6.43(.84)$ & $6.51(.87)$ & .09 & 1.2 & .721 \\
Hippocampus & $1.15(.59)$ & $1.12(.48)$ & .04 & 2.6 & .861 \\
Amygdala & $.37(.2)$ & $.41(.21)$ & .23 & 10.8 & .118 \\
Nucleus accumbens & $.08(.09)$ & $.08(.11)$ & .02 & 2.9 & .630 \\
Red nucleus & $.02(.02)$ & $.03(.02)$ & .18 & 15.2 & .255 \\
Substantia nigra & $.07(.03)$ & $.07(.03)$ & .12 & 5.9 & .595 \\
Pulvinar & $.09(.05)$ & $.11(.04)$ & .4 & 19.6 & .045 \\
\hline
\end{tabular}

Note:-All volumes are reported in milliliters. Differences between groups were tested using the Mann-Whitney $U$ test.

Table 4: Differences in the SDGM normalized volumes between patients with CIS and HC

\begin{tabular}{lcccccc}
\hline & \multicolumn{2}{c}{ Volume } & & \multicolumn{2}{c}{$\%$} & $P$ \\
\cline { 2 - 4 } & HC & CIS & & $d$ & Difference & Value \\
\hline Total SDGM & $45.22(4.59)$ & $45.43(4.1)$ & .05 & .5 & .818 \\
Caudate & $6.75(.99)$ & $6.89(.89)$ & .16 & 2.1 & .437 \\
Putamen & $9.59(1.22)$ & $9.7(1.1)$ & .1 & 1.1 & .617 \\
Globus pallidus & $3.52(.4)$ & $3.44(.35)$ & .2 & -2.3 & .329 \\
Thalamus & $15.21(1.54)$ & $15(1.53)$ & .12 & -1.4 & .550 \\
Hippocampus & $7(.86)$ & $7.16(.74)$ & .2 & 2.3 & .324 \\
Amygdala & $2.38(.36)$ & $2.38(.35)$ & .01 & 0 & .963 \\
Nucleus accumbens & $.79(.18)$ & $.82(.17)$ & .18 & 3.8 & .400 \\
Red nucleus & $.17(.02)$ & $.17(.03)$ & .04 & 0 & .870 \\
Substantia nigra & $.29(.05)$ & $.32(.05)$ & .6 & 10.3 & .003 \\
Pulvinar & $.43(.07)$ & $.44(.07)$ & .09 & 2.3 & .619 \\
\hline
\end{tabular}

Note:-All volumes are reported in milliliters. Differences between groups were tested using the Student $t$ test.

Table 5: Differences in the NPTV between patients with CIS and HC

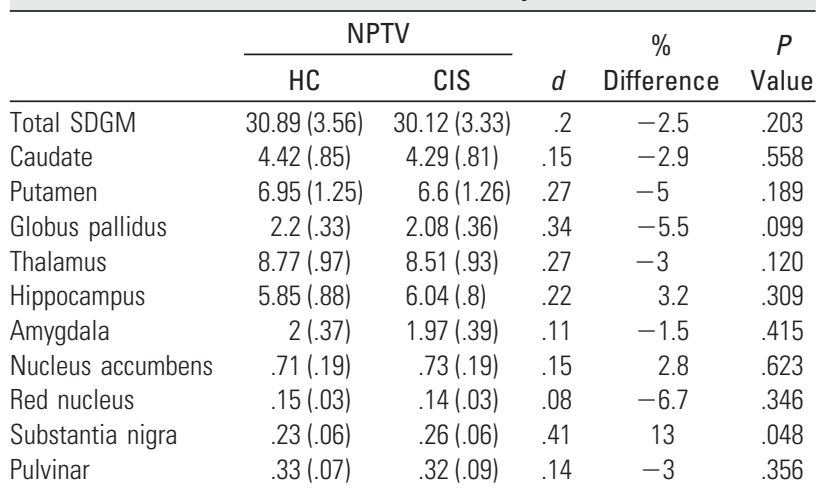

Note:-All volumes are reported in milliliters. Differences between groups were tested using the Mann-Whitney $U$ test.

Table 3 shows differences in MP-APT volume between patients with CIS and HC. A significantly increased volume of the MP-APT was observed in patients with CIS compared with HC in the putamen $(P=.004)$, caudate nucleus $(P=.035)$, pulvinar nucleus of the thalamus $(P=.045)$, and total SDGM $(P=.048)$.

Tables 4 and 5 show normalized volume and NPTV differences between patients with CIS and HC. No significant differences between the study groups were detected. The substantia nigra was significantly larger among patients with 
CIS, compared with $\mathrm{HC}(P=.003)$, and had higher NPTV $(P=.048)$.

To investigate the differences in patients with CIS, they were further divided into subgroups according to median T2LV, T2 lesion number ( $\geq 9$ and $<9$ ), polysymptomatic versus monosymptomatic onset, different subtypes of monosymptomatic onset, and therapy status. No significant differences were found for any of these comparisons.

Increased MP-APT volume of the thalamus was related to increased T2-LV $(P=.033)$. Furthermore, significant correlations were observed between the decreased NWMV measurements, with increased MP-APT volume in total SDGM, caudate, thalamus, hippocampus, and substantia nigra $(P<$ $.05)$. Decreased SDGM volume measurements in total SDGM, caudate nucleus, putamen, globus pallidus, thalamus, hippocampus, and red nucleus volume yielded significant relationships with decreased NWMV $(P<.01)$. Moreover, increased T2-LV was inversely related to the decreased volume of the thalamus and the pulvinar nucleus of the thalamus $(P<$ .05). Decreased NPTV of total SDGM, thalamus, the pulvinar nucleus of the thalamus, and hippocampus was also strongly related to decreased NWMV $(P<.01)$.

No significant relationship was found between increased MP-APT or MP-APT volumes and decreased normalized volumes in the same individual SDGM structures.

\section{Discussion}

In this study, we investigated the extent of iron deposition using SWI-filtered phase measurements in the SDGM of patients with CIS and HC. We observed increased abnormal phase and abnormal phase volume in the SDGM of patients with CIS, compared with age- and sex-matched HC. Significant increases were observed in the total SDGM, caudate nucleus, putamen, and pulvinar nucleus of the thalamus. However, no differences were detected in volumetric measures of the same regions between the 2 study groups. These findings suggest that increased iron deposition in the SDGM structures may precede structure-specific atrophy development.

Iron deposition in the brain parenchyma of patients with MS has been observed using histopathologic techniques. ${ }^{24,25}$ More recently, MR imaging techniques, such as T2 relaxometry, ${ }^{17-19}$ T2 hypointensity, ${ }^{14-16,30,39}$ magnetic field correlation, ${ }^{20}$ and SWI ${ }^{21-23,40}$ have been used to quantify and visualize iron deposition in vivo. Looking at changes in T2 intensity is a relatively straightforward method of evaluating iron content, because increased levels of iron result in reduced T2 relaxation time, leading to hypointensity on T2-weighted images. ${ }^{41}$ In MS, SDGM T2 hypointensity was found to be a predictor of clinical progression, ${ }^{30}$ ambulatory impairment, ${ }^{16}$ cognitive impairment, ${ }^{15,19}$ and brain atrophy. ${ }^{14,19,42,43}$ SDGM T2 hypointensity has also been observed in patients with benign MS. ${ }^{44}$ There is increasing evidence that iron deposition is also present in early stages of MS, such as in patients with pediatric MS and CIS. ${ }^{29,45}$ Ceccarelli et $\mathrm{al}^{29}$ reported T2 hypointensity in the head of the left caudate nucleus of patients with CIS compared with HC. Moreover, atrophy of SDGM structures has also been observed in patients with CIS. ${ }^{3-10}$ Using $\mathrm{R} 2{ }^{*}$ relaxometry, Khalil et $\mathrm{al}^{18}$ found significantly increased relaxation rates, indicative of iron deposition, in the caudate nucleus, putamen, and globus pallidus of patients with RRMS compared with patients with CIS. In their most recent work, ${ }^{19}$ these authors reported that patients with CIS did not present with increased iron content compared with HC. However, the HC were approximately 3 years older than patients with CIS, who presented with much larger variance in age range compared with $\mathrm{HC}$. The approach used in that study did not eliminate all those voxels classified as iron that were expected to be present in healthy subjects in relation to their aging process, with the highest content being in the globus pallidus, putamen, and caudate. ${ }^{23}$ Differences in findings between our study and the latter one on iron content could be related to the sample size (we had twice the number of $\mathrm{HC}$ with lower age variance), use of different iron-based imaging techniques (SWI phase versus relaxometry), or detection of only abnormal iron content, when corrected for age. In contrast to these recent findings, ${ }^{19}$ our results suggest that excessive iron deposition is already present in patients with CIS and increases with disease progression. ${ }^{30,39}$

In this study, we used an SWI-filtered phase approach, which has been histopathologically validated. ${ }^{46}$ SWI is an MR imaging technique capable of visualizing tissues affected by iron deposition in the form of ferritin, deoxyhemoglobin, or hemosiderin. ${ }^{31,47}$ This method was recently used in a study finding strong iron content differences between patients with MS and HC in SDGM. ${ }^{23}$ An SWI-filtered phase approach has several methodologic advantages over T2 hypointensity and relaxometry measurements, being more sensitive to detect tissue changes indicative of iron. . $^{2,31,47}$

Iron deposition may be derived from myelin and oligodendrocyte debris, as well as iron stores in macrophages, or it may be the product of hemorrhages from damaged brain vessels. ${ }^{48}$ Excessive iron may lead to the formation of hydroxyl radicals through Fenton and Haber-Weiss reactions, causing tissue damage. ${ }^{27}$ The measurement of MP-APT allows for the quantification of only the phase values lower than 2 standard deviations below the mean of a previously defined representative control group. ${ }^{23}$ Furthermore, we defined NPTV as brain tissue free of abnormal phase, which may reflect a healthier state of tissue. NPTV was derived by subtracting the abnormal phase tissue volume from the total volume of that same SDGM structure. Recently, robust differences were observed in NPTV of the SDGM using an adult sample of patients with MS compared with HC. ${ }^{23}$ In this study, only a trend for a decrease of NPTV was observed in the globus pallidus. The significant increase in NPTV of the substantia nigra may be a result of increased statistical power to detect significance because this SDGM structure is known to have intrinsically high iron content. $^{49}$

It was recently reported that iron deposition is dominant in the pulvinar nucleus of the thalamus in a sample of adult patients with MS aged 19-35 years. ${ }^{50}$ In addition to changes in the basal ganglia, we also found significant increases of pulvinar MP-APT and MP-APT volume, suggesting that this structure may be exceptionally prone to iron accumulation in MS. Because iron accumulation is observed in the pulvinar nucleus of the thalamus in patients with CIS, it stands to reason that it may be one of the earliest affected SDGM structures.

An important finding of our study was that, with exception of the substantia nigra, no SDGM volume loss was observed in patients with CIS. Moreover, global and region-specific vol- 
ume measurements were also similar between the 2 study groups. In contrast, significant increases of MP-APT and MPAPT volume were observed in the SDGM of patients with CIS. Until now, it has been unclear whether iron deposition is merely an epiphenomenon of MS pathology or if it is an instigator of inflammation and disease development. ${ }^{19,51}$ In a recent study, Williams et al investigated the relationship between inflammation and iron deposition using an original animal model labeled as "cerebral experimental autoimmune encephalomyelitis," which develops CNS perivascular iron deposits. ${ }^{52}$ They reported that inflammatory cell infiltrates were associated with perivascular iron deposits; however, inflammatory cells were also observed without associated iron deposits. There was an association between T2-LV accumulation and increased MP-APT volume and decreased normalized volumes and NPTV in various SDGM structures, especially the thalamus and pulvinar nucleus of thalamus. Therefore, this study provides novel information indicating that iron deposition and atrophy development in basal ganglia are closely related to inflammation, as measured by T2 and Gd lesion accumulation.

The findings from this study also suggest that iron deposition may occur before detectable atrophy in the same structures. In fact, no significant relationship was found between increased MP-APT or MP-APT volumes and decreased normalized volumes in the individual SDGM structures. A previous study showed a direct relationship between increased MPAPT and structure-specific atrophy in different SDGM regions in the later stages of the disease. ${ }^{23}$ Therefore, future longitudinal studies should explore the interplay between iron deposition and atrophy development in individual SDGM structures from the first clinical onset.

Increased MP-APT, MP-APT volume, and decreased normalized volumes and NPTV in total SDGM, caudate, thalamus, pulvinar nucleus of the thalamus, putamen, globus pallidus, hippocampus, substantia nigra, and red nucleus were related with decreased NWMV but not with NGMV. These results indicate that there is a close relationship between iron deposition and WM, but not cortical GM atrophy development at the earliest stages of MS. These findings indicate that global GM atrophy may be preceded by inflammation and iron accumulation that is confined to SDGM and WM regions.

There are several potential limitations inherent in SWIfiltered phase imaging. Phase changes could be the result of changes to the underlying tissue structure or local iron distribution rather than absolute changes in iron content, and different chemical forms of iron could potentially have different SWI-phase effects. ${ }^{53}$ Patients with CIS were relatively older and had somewhat longer disease duration than those enrolled in typical clinical trials. ${ }^{54}$ Presumably, this was because some of the patients with CIS in our study were prospectively enrolled at the time of their referral to our MS specialty center. This could explain the somewhat higher disease duration than reported in clinical trials. ${ }^{54}$ However, clinical and MR imaging features of the enrolled sample of patients with CIS are representative because the observed number and volume of T2 lesions and clinical disability are similar to previously reported baseline CIS cohorts. ${ }^{54}$ In addition, the patients with CIS were approximately 2 years younger than HC. Nevertheless, future studies should investigate iron deposition in younger patients with CIS.

However, we believe that by using a large sample, and data both on abnormal phase and atrophy measures, our study provides support for the concept that iron deposition is present at the earliest MS disease stages and may contribute to disease development and brain damage. As has been shown previously in patients with $\mathrm{MS},{ }^{23}$ the pulvinar nucleus of the thalamus, as well as the basal ganglia, appear to exhibit the most widespread iron deposition in patients with CIS. Future prospective studies should also explore the relationship between the rate of iron deposition and conversion to clinically definite MS and development of disability in patients with CIS.

Disclosures: Bianca Weinstock-Guttman received personal compensation for consulting, speaking, and serving on a scientific advisory board for Biogen Idec, Teva Neuroscience, and EMD Serono. Dr. Weinstock-Guttman also received financial support for research activities from NMSS, NIH, ITN, Teva Neuroscience, Biogen Idec, EMD Serono, and Aspreva. Additional ICMJE Disclosures: UNRELATED: Consultancy: Pfizer, Acorda, Questcor; Grants/Grants Pending: ITN, Novartis, Shire; Payment for Lectures (including service on speakers bureaus): Biogen Idec, Teva Neuroscience, EMD Serono, Pfizer, Acorda.) David Hojnacki has received speaker honoraria and consultant fees from Biogen Idec, Teva Pharmaceutical Industries Ltd., EMD Serono, Pfizer Inc, and Novartis. Robert Zivadinov received personal compensation from Teva Neuroscience, Biogen Idec, EMD Serono and Questcor Pharmaceuticals for speaking and consultant fees. Dr. Zivadinov received financial support for research activities from Biogen Idec, Teva Neuroscience, Genzyme, Bracco, Questcor Pharmaceuticals and EMD Serono.

\section{Acknowledgments}

The authors thank Eve Salczynski for technical assistance in the preparation of this manuscript.

\section{References}

1. Pirko I, Lucchinetti CF, Sriram S, et al. Gray matter involvement in multiple sclerosis. Neurology 2007;68:634-42

2. Fisher E, Lee JC, Nakamura K, et al. Gray matter atrophy in multiple sclerosis: a longitudinal study. Ann Neurol 2008;64:255-65

3. Audoin B, Ibarrola D, Malikova I, et al. Onset and underpinnings of white matter atrophy at the very early stage of multiple sclerosis-a two-year longitudinal MRI/MRSI study of corpus callosum. Mult Scler 2007;13:41-51

4. Audoin B, Zaaraoui W, Reuter F, et al. Atrophy mainly affects the limbic system and the deep grey matter at the first stage of multiple sclerosis. J Neurol Neurosurg Psychiatry 2010;81:690-95

5. Calabrese M, Atzori M, Bernardi V, et al. Cortical atrophy is relevant in multiple sclerosis at clinical onset. J Neurol 2007;254:1212-20

6. Henry RG, Shieh M, Amirbekian B, et al. Connecting white matter injury and thalamic atrophy in clinically isolated syndromes. J Neurol Sci 2009;282:61-66

7. Henry RG, Shieh M, Okuda DT, et al. Regional grey matter atrophy in clinically isolated syndromes at presentation. J Neurol Neurosurg Psychiatry 2008;79:1236-44

8. Ramasamy DP, Benedict RH, Cox JL, et al. Extent of cerebellum, subcortical and cortical atrophy in patients with MS: a case-control study. J Neurol Sci 2009;282:47-54

9. Roosendaal SD, Bendfeldt K, Vrenken H, et al. Grey matter volume in a large cohort of MS patients: relation to MRI parameters and disability. Mult Scler 2011;17:1098-106

10. Fisniku LK, Chard DT, Jackson JS, et al. Gray matter atrophy is related to long-term disability in multiple sclerosis. Ann Neurol 2008;64:247-54

11. Calabrese M, Rinaldi F, Mattisi I, et al. The predictive value of gray matter atrophy in clinically isolated syndromes. Neurology 2011;77:257-63

12. Dalton CM, Chard DT, Davies GR, et al. Early development of multiple sclerosis is associated with progressive grey matter atrophy in patients presenting with clinically isolated syndromes. Brain 2004;127:1101-07

13. Raz E, Cercignani M, Sbardella E, et al. Gray- and white-matter changes 1 year after first clinical episode of multiple sclerosis: MR imaging. Radiology 2010;257:448-54

14. Bakshi R, Benedict RHB, Bermel RA, et al. T2 hypointensity in the deep gray matter of patients with multiple sclerosis: a quantitative magnetic resonance imaging study. Arch Neurol 2002;59:62

15. Brass SD, Benedict RHB, Weinstock-Guttman B, et al. Cognitive impairment is 
associated with subcortical magnetic resonance imaging grey matter T2 hypointensity in multiple sclerosis. Mult Scler 2006;12:437

16. Tjoa CW, Benedict RH, Weinstock-Guttman B, et al. MRI T2 hypointensity of the dentate nucleus is related to ambulatory impairment in multiple sclerosis. J Neurol Sci 2005;234:17-24

17. Burgetova A, Seidl Z, Krasensky J, et al. Multiple sclerosis and the accumulation of iron in the basal ganglia: quantitative assessment of brain iron using MRI t (2) relaxometry. Eur Neurol 2010;63:136-43

18. Khalil M, Enzinger C, Langkammer C, et al. Quantitative assessment of brain iron by $\mathrm{R}(2)^{*}$ relaxometry in patients with clinically isolated syndrome and relapsing-remitting multiple sclerosis. Mult Scler 2009;15:1048-54

19. Khalil M, Langkammer C, Ropele S, et al. Determinants of brain iron in multiple sclerosis: A quantitative 3T MRI study. Neurology 2011;77:1691-97

20. Ge $\mathrm{Y}$, Jensen $\mathrm{JH}, \mathrm{Lu} \mathrm{H}$, et al. Quantitative assessment of iron accumulation in the deep gray matter of multiple sclerosis by magnetic field correlation imaging. AJNR Am J Neuroradiol 2007;28:1639

21. Eissa A, Lebel RM, Korzan JR, et al. Detecting lesions in multiple sclerosis at 4.7 Tesla using phase susceptibility-weighting and T2-weighting. J Magn Reson Imaging 2009;30:737-42

22. Haacke EM, Makki M, Ge Y, et al. Characterizing iron deposition in multiple sclerosis lesions using susceptibility weighted imaging. J Magn Reson Imaging 2009;29:537-44

23. Zivadinov R, Heininen-Brown M, Schirda C, et al. Abnormal subcortical deepgray matter susceptibility-weighted imaging filtered phase measurements in patients with multiple sclerosis. A case-control study. Neuroimage 2012; 59:331-39

24. Craelius W, Migdal MW, Luessenhop CP, et al. Iron deposits surrounding multiple sclerosis plaques. Arch Pathol Lab Med 1982;106:397-99

25. LeVine SM. Iron deposits in multiple sclerosis and Alzheimer's disease brains. Brain Res 1997;760:298-303

26. Fenton H.J.H. Oxidation of tartaric acid in presence of iron. J Chem Soc 1894;65:899-903

27. Khalil M, Teunissen $\mathrm{C}$, and Langkammer C. Iron and neurodegeneration in multiple sclerosis. Mult Scler Int 2011:606807

28. Miller D, Barkhof F, Montalban X, et al. Clinically isolated syndromes suggestive of multiple sclerosis, part I: natural history, pathogenesis, diagnosis, and prognosis. Lancet Neurol 2005;4:281-88

29. Ceccarelli A, Rocca MA, Neema M, et al. Deep gray matter T2 hypointensity is present in patients with clinically isolated syndromes suggestive of multiple sclerosis. Mult Scler 2010;16:39-44

30. Neema M, Arora A, Healy BC, et al. Deep gray matter involvement on brain MRI scans is associated with clinical progression in multiple sclerosis. $\mathrm{J} \mathrm{Neu}$ roimaging 2009;19:3-8

31. Haacke EM, Mittal S, Wu Z, et al. Susceptibility-weighted imaging: technical aspects and clinical applications, part 1. AJNR Am J Neuroradiol 2009;30:19

32. Polman CH, Reingold SC, Banwell B, et al. Diagnostic criteria for multiple sclerosis: 2010 revisions to the McDonald criteria. Ann Neurol 2011;69: 292-302

33. Kurtzke JF. Rating neurologic impairment in multiple sclerosis: an Expanded Disability Status Scale (EDSS). Neurology 1983;33:1444-52

34. Patenaude B, Smith SM, Kennedy DN, et al. A Bayesian model of shape and appearance for subcortical brain segmentation. Neuroimage 2011;56:907-22

35. Batista S, Zivadinov R, Hoogs $M$, et al. Basal ganglia, thalamus and neocortical atrophy predicting slowed cognitive processing in multiple sclerosis. J Neurol 2012;259:139-46

36. Yeh EA, Weinstock-Guttman B, Ramanathan M, et al. Magnetic resonance imaging characteristics of children and adults with paediatric-onset multiple sclerosis. Brain 2009;132:3392-400

37. Zivadinov R, Weinstock-Guttman B, Benedict R, et al. Preservation of gray matter volume in multiple sclerosis patients with the Met allele of the rs6265 (Val66Met) SNP of brain-derived neurotrophic factor. Hum Mol Genet 2007;16:2659-68

38. Zivadinov R, Rudick RA, De Masi R, et al. Effects of IV methylprednisolone on brain atrophy in relapsing-remitting MS. Neurology 2001;57:1239-47

39. Bakshi R, Shaikh ZA, Janardhan V. MRI T2 shortening ("black T2") in multiple sclerosis: frequency, location, and clinical correlation. Neuroreport 2000; 11:15-21

40. Hammond KE, Metcalf $\mathrm{M}$, Carvajal $\mathrm{L}$, et al. Quantitative in vivo magnetic resonance imaging of multiple sclerosis at 7 Tesla with sensitivity to iron. Ann Neurol 2008;64:707-13

41. Drayer B, Burger P, Darwin R, et al. MRI of brain iron. AJR Am J Roentgenol 1986;147:103-10

42. Bakshi R, Dmochowski J, Shaikh ZA, et al. Gray matter T2 hypointensity is related to plaques and atrophy in the brains of multiple sclerosis patients. J Neurol Sci 2001;185:19-26

43. Bermel RA, Puli SR, Rudick RA, et al. Prediction of longitudinal brain atrophy in multiple sclerosis by gray matter magnetic resonance imaging $\mathrm{T} 2$ hypointensity. Arch Neurol 2005;62:1371-76

44. Ceccarelli A, Filippi M, Neema M, et al. T2 hypointensity in the deep gray matter of patients with benign multiple sclerosis. Mult Scler 2009;15:678 - 86

45. Ceccarelli A, Rocca MA, Perego E, et al. Deep grey matter T2 hypo-intensity in patients with paediatric multiple sclerosis. Mult Scler 2011;17:702-07

46. Hopp K, Popescu BF, McCrea RP, et al. Brain iron detected by SWI high pass filtered phase calibrated with synchrotron X-ray fluorescence. J Magn Reson Iimaging 2010;31:1346-54

47. Haacke EM, Xu Y, Cheng YC, et al. Susceptibility weighted imaging (SWI). Magn Reson Med 2004;52:612-18

48. Stankiewicz J, Panter SS, Neema M, et al. Iron in chronic brain disorders: imaging and neurotherapeutic implications. Neurotherapeutics 2007;4:371-86

49. Snyder AM, Connor JR. Iron, the substantia nigra and related neurological disorders. Biochim Biophys Acta 2009;1790:606-14

50. Haacke EM, Garbern J, Miao Y, et al. Iron stores and cerebral veins in MS studied by susceptibility weighted imaging. Int Angiol 2010;29:149-57

51. Zivadinov R, Weinstock-Guttman B, Pirko I. Iron deposition and inflammation in multiple sclerosis. Which one comes first? BMC Neurosci 2011;12:60

52. Williams R, Rohr AM, Wang WT, et al. Iron deposition is independent of cellular inflammation in a cerebral model of multiple sclerosis. $B M C N e u r o s c i$ 2011;12:59

53. Schweser F, Deistung A, Lehr BW, et al. Quantitative imaging of intrinsic magnetic tissue properties using MRI signal phase: An approach to in vivo brain iron metabolism? Neuroimage 2011;54:2789-807

54. Kappos L, Freedman MS, Polman CH, et al. Long-term effect of early treatmen with interferon beta-1b after a first clinical event suggestive of multiple sclerosis: 5-year active treatment extension of the phase 3 BENEFIT trial. Lancet Neurol 2009;8:987-97 